\title{
Pancreatic neuroendocrine tumor in an individual with Von Hippel Lindau syndrome. A case report and review literature
}

\author{
Sandip Ganguly1, Linu A Jacob1, Siddhartha Biswas², KC Lakshmaiah

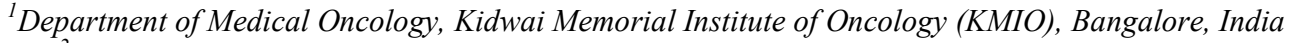 \\ ${ }^{2}$ Department of Pathology, Kidwai Memorial Institute of Oncology (KMIO), Bangalore, India
}

Received March 18, 2015; Revised September 20, 2015; Accepted September 21, 2015; Published Online November 22, 2015

\section{Case Report}

\begin{abstract}
Von Hippel Lindau (VHL) disease is a heritable cancer syndrome characterized by VHL gene mutation in 3p chromosome. Mutations occur at the exons. Rarely mutations at introns have been reported. It leads to accumulation of Hypoxic ischemic factor $\alpha$ (HIF $\alpha)$ which in turn leads to uncontrolled cellular proliferation. Few reports of pancreatic neuroendocrine tumors (PNETs) are there in the literature. Most of them are associated with a mutation in the exon of VHL gene. Association with intron mutation is not there. Here we are reporting a case of PNET in a patient who has a simultaneous mutation both at the exon and the intron.
\end{abstract}

Keywords: Pancreatic Neuro Endocrine; Von-Hippel Lindau; Sunitinib

\section{Introduction}

Gastro-enteropancreatic neuroendocrine tumors arise from the neuroendocrine cells which are present diffusely in the gastrointestinal tract. ${ }^{1}$ The cell type of the tumor is heterogeneousin secreting various types of hormones or being non-secreting at all.

On the basis of presence or absence of clinical syndrome due to hormonal secretion, they are classified as functional or non-functional. ${ }^{2}$ PNETs are rare pancreatic neoplasm with unique tumor biology, presentation, and natural history in contrast to the exocrine counterparts. PNETs occur most commonly as sporadic but they can occur as a component of specific genetic syndrome. Genetic syndromes which are associated with PNETs are Multiple Endocrine Neoplasia 1 (MEN1), Von Hippel Lindau disease (VHL), Neurofibromatosis 1 and Tuberous sclerosis. ${ }^{3}$

\section{Case presentation}

A 19 year old female presented to our outpatient department with history of abdominal pain of 2 weeks duration. There was associated history of early satiety. She was born of a third degree consanguineous marriage. Three of her siblings and her father had died due to cardiovascular diseases in their second decade.
On examination she was found to have stage 1 blood pressure according to Joint National Committee 8 with grade 1 hypertensive retinopathy. Rest of the systemic examination was within normal limits. Hemogram and biochemistry were essentially normal. Ultrasound abdomen revealed a mass in the head of the pancreas. CT scan abdomen revealed mass in the head of the pancreas which was hyperintense on contrast study with a similar mass in the right adrenal gland (Figure 1 - 2). Urinary metanephrine levels were normal. I'131 MIBG scan showed no abnormal uptake in the adrenal glands. She was started on antihypertensive medications. An exploratory laparotomy was planned after the control of blood pressure. Intra operatively the mass was found to be locally advanced with extension to adrenals and surrounding structure.

Biopsy from the mass was taken which revealed WHO grade 2 neuroendocrine tumors (Figure 3 - 6). In view of family history of sudden deaths and presence of pancreatic mass, genetic testing for VHL mutation was done after genetic counseling. Her mother refused to undergo the genetic testing. The test results came positive for VHL gene mutation both at exon3 and intron 1. MRI of the brain showed no abnormality. She was started on tablet Sunitinib. 


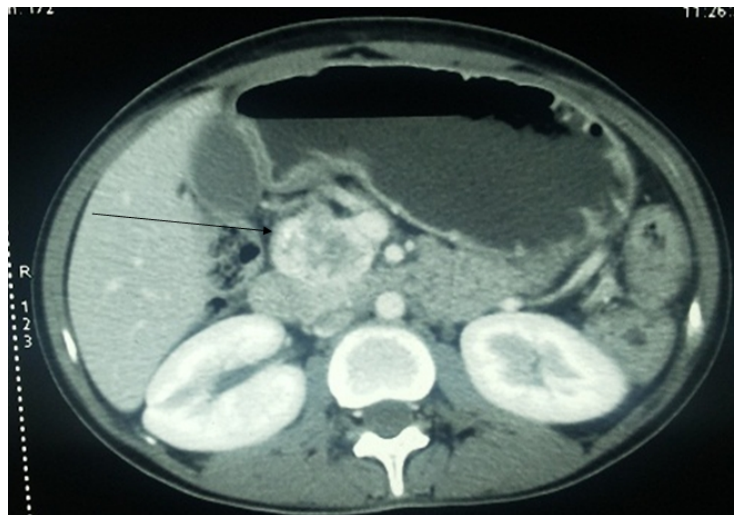

FIG. 1: Ct scan showing a round irregular hyperenhancing lesion at the head of the pancreas.

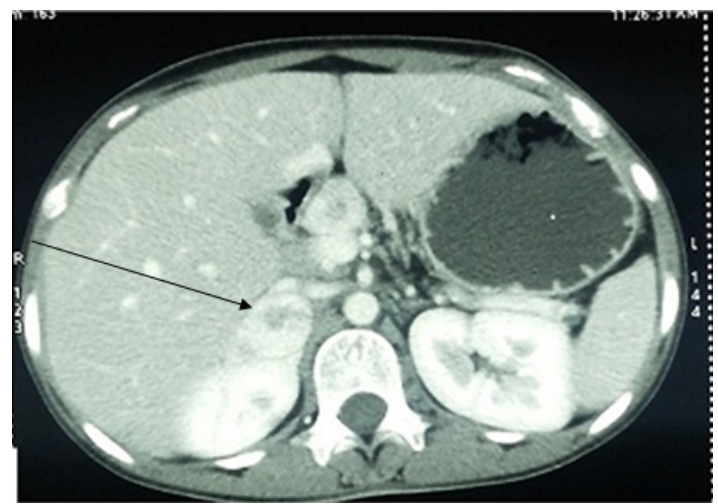

FIG. 2: Ct scan showing round hyperenhancing lesion in the right adrena 1 gland.

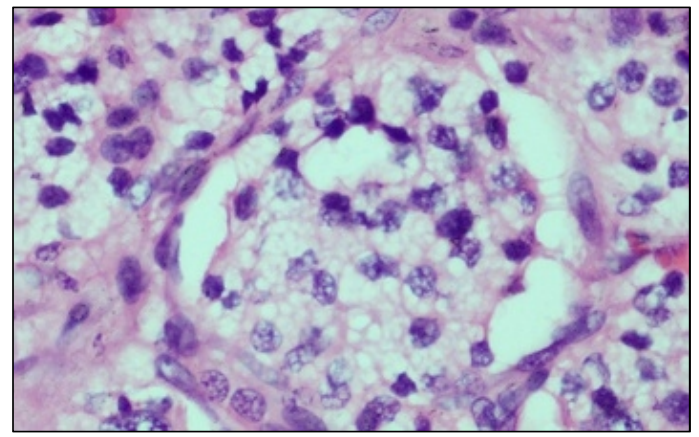

FIG. 3: Small round cells arranged in clusters. No polymorphism is noted.

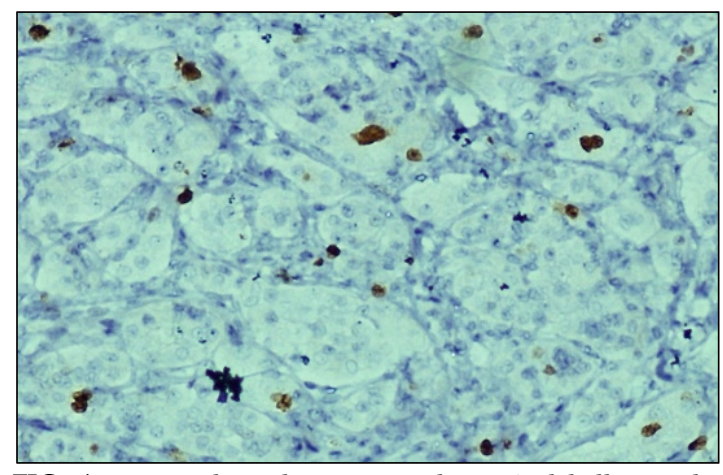

FIG. 4: Immunohistochemistry with Ki 67 labelling index shows positivity with less than $20 \%$.

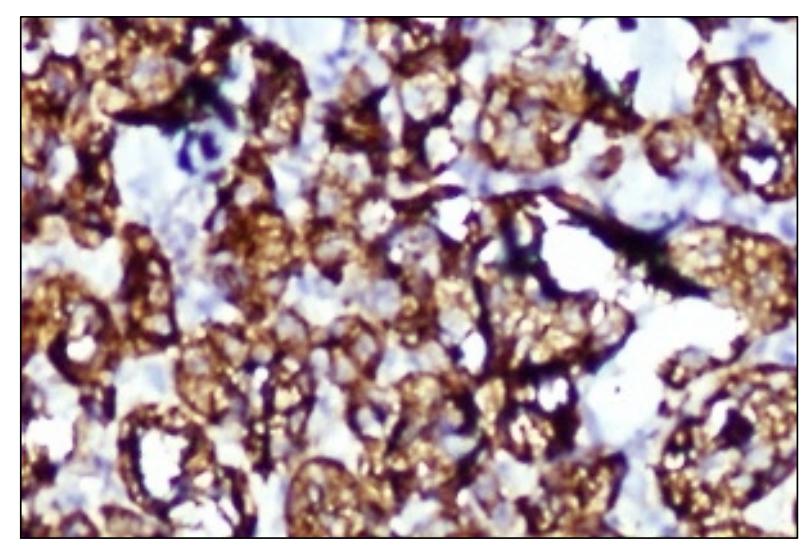

FIG. 5: Immunohistochemistry with chromatogrannin shows positive staining.

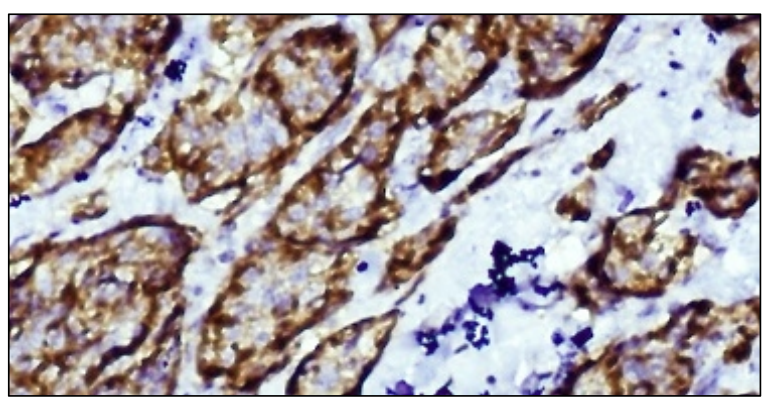

FIG. 6: Immunohistochemistry with synaptophysin shows positive staining.

\section{Discussions}

VHL disease is a heritable genetic disorder with incidence of 1 in 36,000 live births. $80 \%$ of the cases are heritable in an autosomal dominant pattern, whereas the remaining cases are acquired in a sporadic fashion. ${ }^{4}$ The syndrome is characterized by the presence of either a single or combination of tumors like cerebellar hemangioblastoma, retinal angiomas, clear cell carcinomas of kidney, pancreatic endocrine cysts and tumors, pheochromocytomas, and rarely small cell carcinoma of lung and mesothelioma. On the basis of clinical manifestation, VHL syndrome is classified as in Table 1.

The VHL gene was first discovered in 1988 on short arm of chromosome 3 (3p26-p25). Latiff et al., cloned the gene with the establishment of its tumor suppressor activity in $1993 .{ }^{5}$ There is either somatic inactivation of the wild type of allele or loss of heterozygosity of VHL gene or any of the downstream genes which leads to disruption of the normal function of the gene. VHL plays an important role in the oxygen sensing pathway of the cell. ${ }^{6}$ The pathogenesis of VHL syndrome is shown in Table 2. 
TABLE 1: Types of VHL syndrome.

\begin{tabular}{l|l}
\hline \hline VHL Type & \multicolumn{1}{c}{ Characteristics } \\
\hline Type 1 & Hemangioblastoma, with pheochromocytoma being rare. \\
Type 2A & Pheochromocytoma with low risk of clear cell carcinoma and hemangioblastoma. \\
Type 2B & Pheochromocytoma with high risk of clear cell carcinoma and hemangioblastoma. \\
Type 2C & Familial pheochromocytoma with no risk of clear cell carcinoma and hemangioblastoma. \\
Type 3 & Chuvas polycythemia. \\
\hline \hline
\end{tabular}

TABLE 2: Pathogenesis of VHL syndrome.

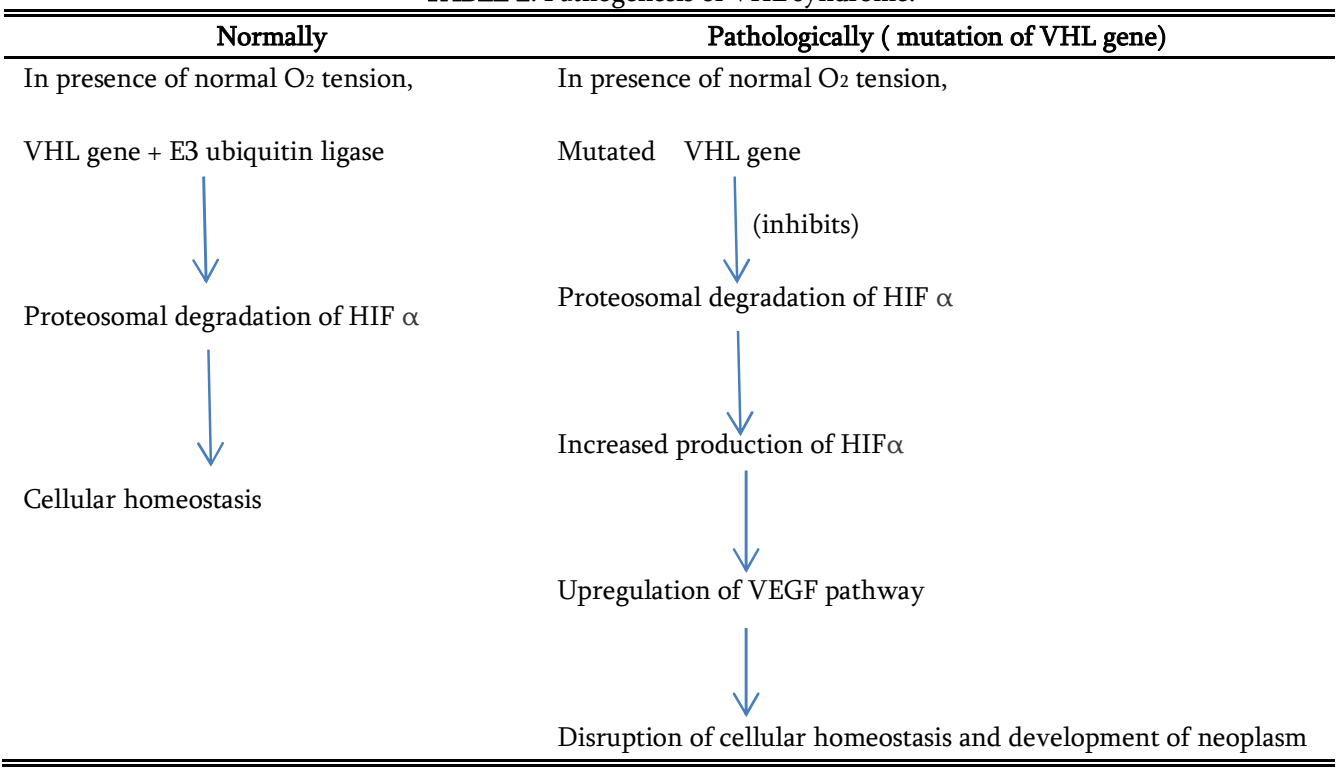

Heterogeneous changes in amino acid residues lead to alteration in the structure and function of protein which in turn influences the disease phenotype. In our patient, there were two heterozygous substitutions. One was a heterozygous substitution of Cytosine to Thymine which lead to change of amino acid Arginine to Tryptophan at codon167 (p.Arg167Trp) in exon 3. This hotspot mutation leads to diminish elongin binding. VHL proteins with disruption of elongin binding are unstable and rapidly degraded by proteasome which ultimately result in tumorigenesis. $^{7} \quad$ Another heterozygous substitution polymorphism was detected in intron 1 of VHL gene. As this intronic variant occurs in splicing donor site, it affects the efficiency of splicing and results in an unspliced transcript. This has been detected in VHL families but its effect on pathogenicity is not known. ${ }^{8}$

Patients can have abnormality in the nervous system, cardiovascular system, adrenals pancreas and even hematological. $77 \%$ of VHL patients can have involvement of pancreas. Pancreatic involvement in VHL is diverse. The most characteristic feature is the presence of pancreatic cyst which includes simple cyst and serous cystadenomas. The cysts don't have any malignant potential. PNETs can be found in $10-15 \%$ of VHL patients. They can occur in isolation or associated with pancreatic cyst. PNETs have malignant potential. ${ }^{9}$
TABLE 3: Grading of Pancreatic neuroendocrine tumor.

\begin{tabular}{l|l}
\hline \hline Grade & \multicolumn{1}{|c}{ Features } \\
\hline Low & $<2$ mitoses/10hpf \& Ki 67 index $<3 \%$ \\
Intermediate & $2-20$ mitoses/10 hpf \& Ki 67 index 3\%-20\% \\
High & $>20$ mitoses/10hpf \& Ki 67 index $>20 \%$ \\
\hline \hline
\end{tabular}

Primary modality of management for PNETs is surgical excision and debulking if possible. There is no role of adjuvant treatment if there is complete surgical excision except in high grade tumors. In surgically inoperable cases, chemotherapy plays a role. The choice of chemotherapeutic agent depends on the grade of the tumor. They are divided into low, intermediate and high grade depending on the mitoses/high power field and Ki67 index as shown in Table 3. ${ }^{10}$ Low and intermediate grade tumors which are inoperable or metastatic targeted therapies like somatostatin analogues like octreotode, mTOR inhibitors like everloimusor tyrosine kinase inhibitors like sunitinib are used. Both everolimus and sunitinb are superior to somatostatin analogues. However, there is no direct comparison between the efficacy of everloimus and sunitinib. High grades are treated by surgery upfront followed by adjuvant chemotherapy, or neoadjuvant chemotherapy followed by surgery. Combination of cisplatin and etoposide is the most common chemotherapeutic agent which is used in high grade variety. In view of grade 2 
inoperable tumor, patient was put on sunitinib and at present she is doing well.

PNETs can have associations with genetic syndromes like VHL. Management of the tumor depends on the surgical operability and the grade of the tumor. There is no role of adjuvant chemotherapy in low and intermediate grade tumors. Targeted therapies like everolimus, sunitinib and octreotide are useful for low and intermediate grade tumors.

\section{Conflict of interest}

The authors declare that they have no conflicts of interest. The authors alone are responsible for the content and writing of the paper.

\section{References}

1. Quaedvlieg PF, Visser O, Lamers CB, et al. Epidemiologyand survival in patients with carcinoid disease in The Netherlands. Anepidemiological study with 2391 patients. Ann Oncol. 2001;12:1295-300.

2. Rindi G, Leiter AB, Kopin AS, et al. The "normal" endocrine cell of the gut: Changing concepts and new evidences. Ann N Y Acad Sci. 2004;1014:1-12.

3. Jensen TR, Berna JM, Bungham BD, Norton AJ. Inherited pancreatic endocrine tumor syndromes: advances in molecular pathogenesis, diagnosis, management and controversies. Cancer. 2008; 113: 1807-43.
4. Lonser RR, Glenn GM, Walther $\mathrm{M}$, et al. von Hippel-Lindau disease. Lancet. 2003;361:2059-67.

5. Latif F, Tory K, Gnarra J, et al. Identification of the von Hippel-Lindau disease tumor suppressor gene. Science. 1993; 260:1317-20.

6. Kim WY, Kaelin WG. Role of VHL gene mutation in human cancer. J Clin Oncol. 2004; 22:4991.

7. Zbar $\mathrm{B}$, Kishida $\mathrm{T}$, Chen $\mathrm{F}$, et al. Germline mutations in the von Hippel- Lindau disease (VHL) gene in families from North America, Europe, and Japan. Hum Mutat. 1996;8:348-57.

8. Asakawa T, Esumi M, Endo S, et al. A mutation at IVS1 +5 of the von Hippel Lindau gene resulting in intron retention in transcripts is not pathogenic in a patient with a tongue cancer. BMC Med Genet. 2012;13:23.

9. Hammel PR, Vilgrain V, Terris B, et al. Pancreatic involvement in von Hippel-Lindau disease. Gastroenterology. 2000;119:1087-95.

10. Klimstra DS, Modlin IR, Coppola D, et al. The pathologic classification of neuroendocrine tumors: a review of nomenclature, grading and staging systems. Pancreas. 2010;39:707-712. 